\title{
Asymptomatic catheter related Rhizobium radiobacter infection in a haploidentical hemapoetic stem cell recipient
}

\author{
Funda Erol Çipe ${ }^{1}$, Figen Doğu ${ }^{1}$, Deniz Sucuoğlu ${ }^{1}$ Derya Aysev², Aydan İkincioğulları \\ ${ }^{1}$ Ankara University, School of Medicine, Department of Pediatric Immunology-Allergy, Ankara, Turkey \\ ${ }^{2}$ Ankara University, School of Medicine, Department of Microbiology, Ankara, Turkey
}

\begin{abstract}
Catheter-related infections are reported as one of the most common sources of nosocomial infections. Rhizobium radiobacter infections manifest with fever and leucocytosis. Here, a 14-month-old girl diagnosed as T (-) B (-) NK (+) severe combined immunodeficiency (SCID) is presented. She had received repeated (x3) unconditioned haploidentical hematopoetic stem cell transplantations. During the follow-up, she was diagnosed with asymptomatic infection with $R$. radiobacter, which was isolated from the central venous catheter and peripheral blood while she was clinically stable, free of symptoms, fever or leucocytosis. She was treated successfully with cefepim and amicasin and did not require catheter removal. This case illustrates the importance of obtaining blood cultures on a regular basis from all patients with an intravascular device, even when they are asymptomatic.
\end{abstract}

J Infect Dev Ctries 2010; 4(8):530-532.

(Received 07 Mar 2010 - Accepted 30 April 2010)

Copyright $\odot 2010$ Cipe et al. This is an open-access article distributed under the Creative Commons Attribution License, which permits unrestricted use, distribution, and reproduction in any medium, provided the original work is properly cited.

\section{Introduction}

Central venous catheters (CVC) are used for patients who require long-lasting procedures such as hematopoetic stem cell transplantation (HSCT). Catheter related blood-stream infections (BSI) are reported as one of the most common sources of nosocomial infections related to Enterobacter, Pseudomonas, Klebsiella, and Stenotrophomonas spp. [1].

Catheter-related Gram-negative bacteremia is diagnosed if one of the following conditions is met: 1) if the catheter culture becomes positive more than two hours before the peripheral blood culture; or 2) if positive cultures of the blood through the CVC and from a peripheral vein positive for the same organism with a colony-forming unit (CFU) ratio (CVC : peripheral blood) of at least 5-10:1 are obtained with no other source for the BSI than the presence of the CVC [1,2].

Formerly known as Agrobium radiobacter, Rhizobium radiobacter is a plant-pathogenic soil inhabitant not characterized as a true human pathogen [3]. It is a small, non-spore forming, Gram-negative, aerobic bacillus [4]. It is an opportunistic pathogen of minor clinical significance and has been reported as a rare cause of bacteremia, endocarditis, and peritonitis, mostly in catheterized immunocompromised patients $[5,6]$. Bacteremia accompanying fever and leukocytosis is the most common manifestation, often occurring without injection site symptoms [7]. Pediatric infections reported by $R$. radiobacter are uncommon. Here we report an asymptomatic CVCrelated bacteremia caused by an $R$. radiobacter strain in a 14-month-old girl who had undergone repeated HSCTs due to severe combined immunodeficiency (SCID).

\section{Case Report}

A 5-month-old girl was referred to our clinic with the diagnosis of T-B-NK+ SCID. A doublelumen subclavian catheter was inserted two weeks prior to her first HSCT. As she did not have a fully matched sibling donor and her clinical condition was unstable, she received peripheral blood stem cell transplantation (PBSCT) without receiving any conditioning regimen from her haploidentical father following CD34+ stem cell selection. As engraftment was not achieved on day 28, CD34+ selected peripheral blood stem cells collected from her mother were reinfused. A third transplantation was performed two months after the second one, due to graft failure. She received conventional doses of busulphan/cyclophosphamide for conditioning and cyclosporine A for graft-versushost disease (GVHD) prophylaxis. Myeloid and platelet engraftments were achieved on days +14 and +18 respectively following the third transplantation. Grade I acute GVHD developed on day +26 and was treated with corticosteroids for ten 
days. She was discharged with co-trimoxazole, acyclovir, fluconazole and intravenous immunoglobulin (IVIG) prophylaxis on day +55 with full donor chimerism.

The patient was admitted to the hospital for her routine clinic visit six months after transplantation. $R$. radiobacter grew from both catheter lumens and also from her peripheral blood sample. $R$. radiobacter identification was performed twice using the API 20NE identification system (bioMérieux, Germany.) for Gram-negative rods. She had no infection-related signs such as fever and there was no evidence of infection from any other source. In addition, there were no physical findings of pain, swelling, erythema or exudate at the CVC insertion site. Intravenous cefepime and amicasin treatments were started emprically. During the antibiotherapy, she had no signs of systemic disease. After 24 hours of the treatment, negative blood cultures were obtained (clear of microorganisms), and treatment was completed in 10 days, without removing the CVC.

\section{Discussion}

There has been an increase in the proportion of nosocomial device-related infections over the years. Prolonged hospital stay and increased mortality were reported with antibiotic resistant Gramnegative organisms, but most studies focused on nosocomial Gram-positive infections [1]. Some authors reported that $R$. radiobacter is spread nosocomially, but other described cases do not support this route [6]. Our patient came to the hospital for a routine control visit and to receive prophylactic IVIG infusion; therefore, soil exposure or recent hospitalization history are not viable as possible sources of the microorganism.

$R$. radiobacter infections related to heparin infusions have been reported in some cases [8,9]. In addition, hospital-acquired Gram-negative bacteremia has been found associated with intravenous administration of heparin solution in the absence of phlebitis [10]. In the light of these reports, we can speculate that heparin that is used for CVC care might be the causative agent of $R$. radiobacter bacteremia in this presented case.

In about $10 \%$ of cases, Gram-negative bacilli develop resistance to any single agent [3]. It has been reported that $R$. radiobacter isolates often showed resistance to tobramycin and ceftazidime. Thus, ciprofloxacin is generally considered the first-line drug [6]. Consistent with previous reports, our susceptibility profile showed that aminoglycosides, third-generation cephalosporins, fluoroquinolones, extended-spectrum beta-lactams, and carbapenems could be used for empirical treatment in our patient. However, though she was asymptomatic, we treated her with a cefepime and amicasin combination to keep the IV access via $\mathrm{CVC}$ as long as possible. The suggested duration of treatment is 10 to 14 days following the sterile blood culture $[7,10]$. Our patient was treated for 10 days.

One prospective study reported that antibiotic treatment was not sufficient to achieve a successful outcome alone, and delayed catheter removal was associated with complicated bacteremia [9]. Prompt catheter removal was significantly associated with an increased chance of survival. The device was removed in more than half of the patients and cure was achieved only after the device was removed $[6,11,12]$. In our patient, response to antibiotherapy was excellent and there was no recurrence even though the CVC was not removed. It was considered that the CVC should be removed only if bacteremia continued despite appropriate antibiotic therapy or if the patient became unstable.

In the literature, $R$. radiobacter infections are most likely associated with the intravascular device [6,13]. Most of the patients have debilitating underlying diseases and immunosupression. In one report, three of 13 microorganisms were isolated from patients who were not immunosuppressed but who had intravascular devices due to other conditions such as celiac sprue, coronary arterial disease, and pregnancy [6]. Although neutrophil and lymphocyte engraftments were achieved successfully and neutrophil and lymphocyte counts were normal at six months following HSCT, our patient can still be considered as immunosuppressed due to the lack of functional reconstitution of a full immune system, which needs at least one year's time to develop following haploidentical HSCT. We therefore believe that the partially immunocompromised status of our patient might have played a role in the development of the CVC infection. However, her successful engraftment allowed her to remain asymptomatic.

In conclusion, we present an infant with unusual presentation of $R$. radiobacter bacteremia following HSCT in whom fast and clear response to antibiotherapy was achieved. This case is of interest because the patient's partial immunosuppressed status and the use of heparin in routine CVC care may have played a role in the development of $R$. radiobacter bacteremia. The patient remained asymptomatic and did not require removal of the CVC due to her successful engraftment and appropriate treatment. 


\section{References}

1. Hanna H, Afif C, Alakech B, Boktour M, Tarrand J, Hachem R, Raad I (2004) Central venous catheter-related bacteremia due to Gram-negative bacilli: Significance of catheter removal in preventing relapse. Infect Control Hosp Epidemiol 25: 646-649.

2. Goede MR, Coopersmith CM (2009) Catheter-related bloodstream infection. Surg Clin N Am 89: 463-474.

3. Rogues AM, Sarlangue J, de Barbeyrac B, Doerman HP, Helmer C, Labadie JC, Delfeau C, Sandler B, Bebear C, Gachie JP (1999) Agrobecterium radiobacter as a cause of pseudobacteremia. Infect Control Hosp Epidemiol 20: 345-347.

4. Chen CY, Hansen KS, Hansen LK (2008) Rhizobium radiobacter as an opportunistic pathogen in central venous catheter-associated bloodstream infection: case report and review. J Hosp Infect 68: 203-207.

5. Namdari H, Hamzavi S, Peairs RR. (2003) Rhizobium (Agrobacterium) radiobacter identified as a cause of chronic endophthalmitis subsequent to cataract extraction. J Clin Microbiol 41: 3998-4000.

6. Lai CC, Teng LJ, Hsueh PR, Yuan A, Tsai KC, Tang JL, Tien HF. (2004) Clinical and microbiological characteristic of Rhizobium radiobacter infections. Clin Infect Dis 38: 149-153.

7. Manfredi R, Nanetti A, Ferri M, Mastroianni A, Coronado OV, Chiodo F (1999) Emerging Gram-negative pathogens in the immunocompromised host: Agrobacterium radiobacter septicemia during HIV disease. New Microbiol 22: 375-82.
8. Peiris K, Gee TM, Boswell TC (2006) Bloodstream infection related to heparin infusion caused by Agrobacterium tumefaciens. J Hosp Infect 62: 250-1.

9. Siegman-Igra Y, Jacobi E, Lang R, Schwartz D, Carmeli Y (2005) Unexpected hospital acquired bacteremia in patients at low risk of blood stream infection: the role of heparin drip. J Hosp Infect 60: 122-128.

10. Benjamin DK Jr, Miller W, Garges W, Benjamin DK, McKinney RE Jr, Cotton M, Fisher RG, Alexander KA.(2001) Bacteremia, central catheters and neonates: when to pull the line. Pediatrics 107: 1272-1276.

11. Paphitou NI, Rolstron KVI (2003) Catheter-related bacteremia caused by Agrobacterium radiobacter in a cancer patient: case report and literature review. Infection 31: 421-424.

12. Alnor D, Frimodt-Moller N, Espersen F, Frederiksen W (1994) Infections with the unusual human pathogens Agrobacterium species and Ochrobactrum anthropi. Clin Infect Dis 18: 14-20.

13. Amaya RA, Edwards MS (2003) Agrobacterium radiobacter bacteremia in pediatric patients: case report and review. Pediatr Infect Dis J 22: 183-186.

\section{Corresponding author}

Prof. Dr. Aydan İkincioğulları

Ankara University, School of Medicine, Department of Pediatric Immunology-Allergy

Cebeci, 06100 Ankara/TURKEY

Phone: +90 312595 6346; Fax: +90 3123191440

Email: aydan@mac.com

Conflict of interest: No conflict of interest is declared. 